\title{
Therapeutic monitoring of psychoactive drugs - antidepressants: A review
}

\author{
Milan Grundmannn, Ivana Kacirova ${ }^{\mathrm{a}, \mathrm{b}}$, Romana Urinovska ${ }^{\mathrm{b}}$
}

\begin{abstract}
Background. Major depression, is one of the most prevalent mental disorders in Europe and the USA. The dramatic rise in pharmacological antidepressants is mainly due to increase in use of selective serotonin reuptake inhibitors, serotonin and norepinephrine reuptake inhibitors and other new generation antidepressants. In clinical practice, optimum individual doses are often guided by trial-and-error. This article reviews the available literature on therapeutic monitoring of antidepressant drugs.

Methods. A search using MEDLINE (english-language reports, 1983 - August 2012) with the key words for antidepressant drugs and therapeutic drug monitoring.

Results. There is a need for monitoring antidepressants due to wide interindividual pharmacokinetic variability. At the same drug dose, a more than 20-fold variation in steady state concentration of drug in the body may result: people differ in their ability to absorb, distribute, metabolise and excrete drugs for reasons of concurrent disease, age, gender, smoking and eating habits, concomitant medication and genetics.

Conclusions. Monitoring of antidepressant drugs enables us to individualise drug doses based on rational therapy, minimalise side effects, reduce morbidity and mortality and cut the cost of health care. Phenotyping and genotyping could increase therapeutic drug monitoring furthere.
\end{abstract}

Key words: therapeutic drug monitoring, antidepressant drugs, therapeutic reference ranges

Received: September 21, 2012; Accepted with revision March 6, 2013; Available online: 21 March, 2013 http://dx.doi.org/10.5507/bp.2013.020

aDepartment of Clinical Pharmacology, Faculty of Medicine, University of Ostrava, Czech Republic
bDepartment of Clinical Pharmacology, University Hospital Ostrava
Corresponding author: Milan Grundmann, e-mail: milan.grundmann@osu.cz

Major depression is one of the most prevalent mental disorders in Europe and the United States, with a $16.5 \%$ and $8.9 \%$ lifetime prevalence for European women and men and $16.2 \%$ and $6.6 \%$ for American women and men. This is the case after more than half a century of modern psychopharmacology, with billions of dollars spent on antidepressants annually world-wide. The dramatic rise in consumption of antidepressants in developed countries in the past two decades has been mainly due to increase in the use of selective serotonin reuptake inhibitors, serotonin and norepinephrine reuptake inhibitors and other new generation antidepressants which are now the most commonly prescribed antidepressants in the world. However, we lack good evidence to guide our everyday decisions in conducting antidepressant treatment of patients with major depression ${ }^{1}$. There are several commonly used classes of antidepressants. These include selective serotonin reuptake inhibitors (SSRIs), serotonin and norepinephrine reuptake inhibiors (SNRIs), atypical antidepressants (eg, bupropion and mirtazapin), and serotonin antagonists and reuptake inhibitors (SARIs). Older classes of antidepressants (tricyclic antidepressants - TCAs and monoamine oxidase inhibitors - MAOIs) are still used occasionally. Therapeutic drug monitoring (TDM) in the field of psychotropic drugs began with the tricyclic antidepressants in the 1960s $\left(\right.$ ref. $\left.^{2}\right)$. Although there is sufficient evidence for the benefits of TDM in optimizing antidepressant therapy, its current use in routine care is far from optimal ${ }^{3}$. In clinical practice, the effort to determine the individual antidepressant drug dose optimum is often guided by a trial-and-error dose titration strategy ${ }^{4}$.

Tricyclic antidepressants and monoamine oxidase inhibitors

With the tricyclic antidepressant drugs, TDM is a longestablished tool for finding the individual dose optimum. TDM has been reported to increase not only efficacy and safety of TCAs, but also cost-effectiveness in the treatment of depression and is highly recommended for most tricyclic antidepressants. It reduces the risk of intoxication, and for many TCAs, a plasma concentration-clinical effectiveness relationship has been shown ${ }^{5-9}$. These older agents are limited by their greater risk of overdose, potential effects on cardiac conduction/arrythmias (TCAs), and substantial restrictions on diet and medications (MAOIs). TCAs are also associated with orthostatic hypotension, sedation, and anticholinergic effects, and MAOIs commonly cause orthostasis as well ${ }^{10}$.

\section{Selective serotonin reuptake inhibitors}

Serotonin (5-hydroxytryptamine) is an ancient chemical, evolving at least one billion years ago, and it is present in fungi, plants, and animals. It belongs to a class of biochemicals called monoamines, which also includes norepinephrine, and dopamine. Many adaptive processes evolved to be regulated by serotonin, including cell differentiation, temperature, platelet activation and the clotting process, digestion and gut movement, insulin, electrolyte balance, astrocytic activity, neuronal apoptosis, cerebral blood flow, emotion, attention, aggression, mood, repro- 
ductive function, and mating behavior. Because serotonin regulates many adaptive processes, antidepressants based on serotonin reuptake inhibition could have many adverse health effects. Antidepressants can cause developmental problems, they have adverse effects on sexual and romantic life, and they increase the risk of hyponatremia, bleeding, stroke, and death in the elderly ${ }^{11}$. The SSRIs are the first-line and the most commonly prescribed antidepressants. All SSRIs share similar pharmacologic actions, including minimal anticholinergic, antihistaminic, and alpha1-adrenergic blocking effects, and potent presynaptic inhibition of serotonin reuptake. They are generally welltolerated and are not as dangerous in overdose as are the older agents ${ }^{10}$. The SSRIs are all lipophilic agents, which are well absorbed via the gut mucosa. Fluoxetine, paroxetine, and especially sertraline are highly blound to plasma proteins (95-98\%), whereas the protein binding is less pronounced for citalopram and fluvoxamine $(\leq 80 \%)$. The drugs are extensively distributed to tissues, with volumes of distributions in the range of 400-3000 L. All of the SSRIs are predominantly eliminated by cytochrome P450 catalyzed oxidation in the liver. Fluoxetine, paroxetine and fluvoxamine have all been shown to saturate their own metabolism resulting in nonlinear kinetics within therapeutic dosing ranges. Hence, higher doses of these drugs produce a disproportionate increase in their plasma concentrations. Consequently, it may be difficult to predict the dose necessary to achieve a certain plasma concentration based on the drug plasma concentrations obtained with another dose. The disproportionate increase in plasma concentrations is particularly important with respect to elderly patients. In contrast, citalopram and sertraline follow linear konetics within their therapeutic relevant ranges $^{12}$. To obtain a drug plasma concentration with any predictive value, one should be sure that a steady-state level of the drug has been achieved, i.e. the first blood sample should be drawn 4-5 elimination half-lives after initiation of therapy. Citalopram, sertraline, paroxetine, and fluvoxamine are eliminated with half-lives of about 12-36 h, so after 7-14 days all patients are expected to be in a steady-state. The elimination half-life of fluoxetine is long: up to 6 days for fluoxetine and 16 days for the active metabolite norfluoxetine (Table 1). Thus, steady state levels of norfluoxetine will not be achieved until approximately 3 months after initiation of fluoxetine therapy ${ }^{12}$. The importance of therapeutic drug monitoring of SSRIs is the subject of controversial discussion. However, TDM offers information about drug interactions, insufficient therapeutic effect, side effects, compliance and overdose ${ }^{13}$. The basic characteristics of SSRIs and other newer classes of antidepressants are presented in Table 1.

Citalopram: This is administered as a racemic mixture, pharmacologically active is S-enantiomer. Its oral bioavailability is $80 \%$, the maximum plasma concentration $\left(\mathrm{C}_{\max }\right)$ is reached in $2-4 \mathrm{~h}$ and is $<80 \%$ bound to plasma proteins. It is metabolized mainly through $\mathrm{N}$-desmethylation in the liver to desmethylcitalopram, didesmethylcitalopram and citalopram-N-oxid (pharmacologically active) primarily by cytochrome P450 (CYP) form 2C19 with additional contributions by CYP3A4 and CYP2D6. Polymorphism of CYP2C19 plays an important role in the $\mathrm{N}$-demethylation of citalopram in vivo. Extensive and poor metabolizers of CYP2C19 caused a significant difference in the behaviour of citalopram ${ }^{14}$. The mean elimination half-life is $33-37 \mathrm{~h}$, steady-state plasma concentrations is reached after 7-14 days of dosing. There is a highly linear correlation between citalopram plasma concentrations and citalopram doses, well above the usual dose range. Patients $>65$ years had significantly higher dose-corrected citalopram plasma concentrations than younger patients ${ }^{15}$. Citalopram is widely used, because it does not exhibit pharmacokinetic interactions unlike fluoxetine, fluvoxamine, or paroxetine. For citalopram one observational study revealed that plas-

Table 1. Basic characteristic of antidepressant drugs $\mathrm{s}^{9,12,48,54}$.

\begin{tabular}{|c|c|c|c|c|c|}
\hline Drug & $\begin{array}{c}\text { Bioavailability } \\
(\%)\end{array}$ & $\begin{array}{c}\text { Protein binding } \\
(\%)\end{array}$ & $\begin{array}{c}\mathrm{T}_{\max } \\
\text { (hours) }\end{array}$ & Half-life (hours) & $\begin{array}{c}\text { Time to reach } \\
\text { steady-state (days) }\end{array}$ \\
\hline $\begin{array}{l}\text { Bupropion } \\
\text { (Hydroxybupropion) }\end{array}$ & $87-100$ & 84 & $3-5$ & $\begin{array}{c}8-26 \\
(17-47)\end{array}$ & 8 \\
\hline Citalopram & 80 & $<80$ & $2-4$ & $33-37$ & $7-14$ \\
\hline Duloxetine & $32-80$ & 96 & $6-10$ & $8-17$ & 5 \\
\hline Escitalopram & 80 & 56 & $3-6$ & $22-32$ & $7-10$ \\
\hline $\begin{array}{l}\text { Fluoxetine } \\
\text { (Norfluoxetine) }\end{array}$ & 70 & 95 & $6-8$ & $\begin{array}{c}\text { 4-6 days } \\
\text { (4-16 days) }\end{array}$ & (3 months) \\
\hline Fluvoxamine & 50 & 80 & $3-8$ & $15-20$ & $5-10$ \\
\hline Mirtazapine & 50 & 85 & 2 & $20-40$ & $4-6$ \\
\hline Paroxetine & $30-60$ & 95 & $3-5$ & $12-44$ & $4-14$ \\
\hline Sertraline & $>44$ & 98 & $4-8$ & $26-36$ & 7 \\
\hline Trazodone & $>60$ & $89-95$ & $1-4$ & $\begin{array}{l}\text { distribution 3-6 } \\
\text { elimination 5-9 }\end{array}$ & $2-3$ \\
\hline $\begin{array}{l}\text { Venlafaxine } \\
\text { (O-desmethylvenlafaxine) }\end{array}$ & $40-45$ & 27 & $1-6$ & $\begin{array}{c}5 \\
(11)\end{array}$ & 3 \\
\hline
\end{tabular}

$\mathrm{T}_{\max }$ - time to reach maximum plasma concentration $\left(\mathrm{C}_{\max }\right)$ 
ma concentrations on day 7 of treatment were predictive for later non-response. Patients with citalopram plasma concentrations below $50 \mathrm{ng} / \mathrm{mL}$ had a significantly reduced effect on the Hamilton rating scale for depression ${ }^{15}$. Recent studies correlated plasma concentrations of SSRIs and occupancy of serotonin transporters (5-HTT) using positron emission tomography in healthy control subjects. Striatal 5-HTT occupancy correlated well with plasma concentrations of the SSRIs, and it was concluded that $80 \%$ occupancy is associated with maximal therapeutic effects after 4 weeks of SSRI administration. For citalopram, it was shown that at least $50 \mathrm{ng} / \mathrm{mL}$ is required to attain 80\% 5-HTT occupancy. Despite therapeutic doses, a significant number of patients had serum concentrations less than $50 \mathrm{ng} / \mathrm{mL}$, and these were associated with an unfavorable treatment outcome; therapeutic drug monitoring is therefore recommended to optimize dosing citalopram in the early phase of treatment ${ }^{16}$.

Escitalopram: It is a S-enantiomer of the racemic selective serotonin reuptake inhibitor antidepressant citalopram. It is rapidly absorbed (oral bioavailability $80 \%$ ), and reaches maximum plasma concentrations in approximately 3-6 h after administration. Escitalopram has low protein binding (56\%) and is widely distributed throughout tissues. The elimination half-life of escitalopram is about 22-32 h, steady-state concentrations are achieved within 7-10 days of administration. Escitalopram is metabolized by the CYP2C19, CYP2D6 and CYP3A4. S-desmethylcitalopram (S-DCT), the principal metabolite, is present at approximately one-third the level of escitalopram; however, S-DCT is a weak inhibitor of serotonin reuptake and does not contribute appreciably to the therapeutic activity of escitalopram. The didesmethyl metabolite of escitalopram is typically present at or below quantifiable concentrations. Escitalopram and S-DCT exhibit linear and dose-proportional pharmacokinetics. Adolescents, elderly individuals and patients with hepatic impairment show no clinically relevant differences in pharmacokinetics compared with healthy young adults, implying that adjustment of the dosage is not necessary in these patient groups ${ }^{17}$. TDM of escitalopram is recommended for dose titration and for special indications or problem solving 9 .

Fluoxetine: This is administered as a racemate of $\mathrm{R}$ and S-fluoxetine with almost equal affinity to the 5-HT reuptake carrier. It is well absorbed after oral intake, is highly protein bound (95\%), and has a large volume of distribution $\left(20-40 \mathrm{~L} / \mathrm{kg}\right.$ ). The $\mathrm{C}_{\max }$ is reached within 6-8 h. Fluoxetine is metabolized primarily via $\mathrm{N}$-demethylation to the active metabolite norfluoxetine primarily by CYP2D6 with additional contributions from CYP2C9, CYP2C19, CYP3A4 and CYP2B6 (ref., ${ }^{918}$ ). The elimination half-life of fluoxetine is about 4-6 days, while that of its metabolite norfluoxetine about 4-16 days. Fluoxetine has a nonlinear pharmacokinetic profile. On the basis of the results of plasma concentration-clinical response relationship studies, there appears to be a therapeutic window for fluoxetine. Concentrations of fluoxetine plus nor- fluoxetine above $500 \mathrm{ng} / \mathrm{mL}$ appear to be associated with a poorer clinical response than lower concentrations ${ }^{19}$.

Fluvoxamine: This is well absorbed after oral intake (oral absorption $\geq 94 \%$ ), first pass metabolism reduces the bioavailability to about $50 \%$. The $\mathrm{C}_{\max }$ is reached within 3-8 h, plasma protein binding is about $80 \%$. Fluvoxamine undergoes complex hepatic metabolism, with main metabolic routes that include oxidative demethylation and oxidative deamination (CYP1A2 and CYP2D6) (ref. ${ }^{18}$ ). Fluvoxamine has an elimination half-life of approximately 15-20 h. It has been shown that the oral clearance decreases with increasing doses, suggesting that fluvoxamine exhibits nonlinear kinetics within the therapeutic dose interval. There is a therapeutic threshold for steady-state plasma concentrations of fluvoxamine and probably also for its major metabolite fluvoxamine acid ${ }^{20}$.

Paroxetine: This is well absorbed from the gastrointestinal tract. It undergoes a partially saturated first pass metabolism which reduces the bioavailability to about $30-60 \%$. Maximal blood levels are reached 3 to $5 \mathrm{~h}$ after administration. Paroxetine is highly protein bound (95\%) (ref. ${ }^{21}$ ). An elimination half-life is $12-44 \mathrm{~h}$, steady-state levels are achieved after 4-14 days. Paroxetine exhibits nonlinear kinetics. It is eliminated after transformation in the liver into pharmacologically inactive metabolites. CYP2D6 (high affinity) and CYP3A4 (low affinity) are most likely to be the major contributors to paroxetine metabolism in humans. For some individuals, CYP1A2 could be of importance for paroxetine metabolism, whereas the importance of CYP2C19 and CYP3A5 is probably limited. Nonlinear paroxetine kinetics is more prominent in extensive metabolizers of CYP2D6 than in poor metabolizers of CYP2D6. Elderly subjects taking paroxetine have higher plasma concentrations and slower elimination than younger subjects ${ }^{22}$. Elimination is also reduced in severe renal and hepatic impairment ${ }^{23}$. Paroxetine is characterized by large interindividual pharmacokinetic variability and heterogeneous response patterns ${ }^{24}$. For paroxetine, a positive correlation was found between drug concentration in plasma and serotonin syndrome symptoms ${ }^{25}$.

Sertraline: The $\mathrm{C}_{\max }$ of sertraline is reached within 4-8 $\mathrm{h}$, plasma protein binding is about $98 \%$. It undergoes extensive first-pass metabolism and has a linear pharmacokinetic profile. Several P450 enzymes have been shown to catalyze sertraline N-demethylation, with CYP2B6 contributing to the greatest extent, with lesser contributions from CYP2C19, CYP2C9, CYP3A4, and CYP2D6. For deamination, data support the role for CYP3A4 and CYP2C19. Purified human monoamine oxidases A and B also catalyzed sertraline deamination. Because it appears that there are multiple enzymes involved in initial metabolic pathways of sertraline, it would be difficult for any single agent to cause a meaningful drug interaction via inhibition of the metabolic clearance of sertraline ${ }^{26}$. The elimination half-life of sertraline is about $26-36 \mathrm{~h}$ and of its main metabolite N-desmethylsertraline between 62 104 h. Steady-state concentrations are achieved within 7 
days of administration. Smokers had significantly lower concentration-to-dose mean ratios of serum sertraline and desmethylsertraline than nonsmokers and higher sertraline and desmethylsertraline concentration-to-dose mean ratios were found in elderly patients than in adults aged less than 65 years. A profound variability was found for the interindividual steady-state and trough serum levels of sertraline and desmethylsertraline, but intraindividual metabolizing stability over time was found. The results highlight sertraline TDM as a tool for individual dose optimization and evaluation of patient drug compliance as well as drug-drug interactions. Knowledge of the concentration of the desmethyl metabolite of sertraline is not required to relate clinical effect with drug concentration. However, in some cases the concentration of desmethylsertraline may be helpful to assess the degree of compliance. No correlation between serum drug concentrations and clinical effect was found for sertraline ${ }^{27-29}$. However, monitoring the sertraline plasma level, even though not strictly necessary from a clinical point of view, can be useful in optimizing treatment ${ }^{30}$.

\section{Serotonin and norepinephrine reuptake inhibiors}

Venlafaxine and duloxetine are the two most commonly used SNRIs. They block the reuptake of serotonin and norepinephrine. These agents are generally well-tolerated and safer than older agents in overdose.

Venlafaxine: It is metabolized primarily by the highly polymorphic cytochrome P450 enzyme CYP2D6 to yield a pharmacologically active metabolite, O-desmethylvenlafaxine (ODV), and to a lesser extent by CYP3A4, to yield $\mathrm{N}$-desmethylvenlafaxine ${ }^{31}$.Venlafaxine (VEN) and ODV are mixed serotonin and norepinephrine reuptake inhibitors, venlafaxine is a moderate inhibitor of dopamine reuptake. Only $40-45 \%$ of the dose reaches the systemic circulation unchanged, because of extensive first-pass metabolism. Venlafaxine and its main metabolite ODV are $27 \%$ and $30 \%$ bound to plasma proteins, respectively. Maximal blood levels are reached 1-2 h (VEN) and $3 \mathrm{~h}$ (ODV) after administration of fast-released form and $6 \mathrm{~h}(\mathrm{VEN})$ and $9 \mathrm{~h}$ (ODV) after administration of slowreleased form. The steady-state half lives of venlafaxine and its active metabolite ODV are 5 and $11 \mathrm{~h}$, respectively. Steady-state plasma concentrations are reached after 3 days of dosing. The ratio of ODV/VEN is an effective means of phenotyping individuals according to their CYP2D6 metabolizer status ${ }^{32}$. Serum levels varied widely at each dose level. Sex, age and smoking should be considered for optimal dosing of patients with VEN. Women had higher dose-corrected serum levels of VEN and ODV than men, and patients older than 60 years showed higher levels of both compounds than younger ones. In smokers, mean serum levels of ODV is lower than in non-smokers ${ }^{33}$. A significant correlation was found between VEN plasma levels and its antidepressant efficacy ${ }^{34}$. By Gex-Fabry et al. earlier clinical response may occur with higher VEN+ODV plasma level ${ }^{35}$. A positive correlation was also found between VEN plasma concentration and adverse effects. Patients with ODV/VEN ratios below
0.3 had more side effects and reduced serum concentrations of sodium in comparison with other patients. A poor metabolizers of CYP2D6 increases the risk of VEN side effects $^{36,37}$.

Duloxetine: This achieves a maximum plasma concentration approximately 6-10 h after dosing. Duloxetine is highly protein bound (96\%) and is widely distributed throughout tissues. Oral bioavailability is approximately $50 \%(32-80 \%)$. It is rapidly and extensively metabolized in the liver by CYP1A2 and CYP2D6, and its numerous metabolites, which are inactive, are mainly excreted in the urine. The mean elimination half-life of duloxetine is approximately 8-17 h $\left(\right.$ ref. $^{38,39}$ ). Therapeutic drug monitoring of duloxetine and titration to steady-state serum concentrations above $58 \mathrm{ng} / \mathrm{mL}$ is useful for treatment optimization $^{40}$.

\section{Atypical antidepressants}

Mirtazapine, a serotonin-receptor blocker that also has effects on norepinephrine via blockade of alpha renergic receptors, and bupropion, an agent with effects on norepinephrine and dopamine, represent important alternatives to the SSRIs and SNRIs and, for treatmentresistant depression, are sometimes used in combination with them in more complex pharmacologic regiments. Bupropion is a potent inhibitor of CYP2D6. Mirtazapine causes significant blockade at histamine $(\mathrm{H} 1)$ receptors and 5-HT2 receptors, leading to sedation and incresed appetit/weight gain.

Mirtazapine: This is a tetracyclic antidepressant, the first noradrenergic and specific serotonergic antidepressant (NaSSA). It is rapidly and well absorbed from the gastrointestinal tract after oral administration, and peak plasma concentrations are reached within $2 \mathrm{~h}$. Mirtazapine binds to plasma proteins $(85 \%)$ in a nonspecific and reversible way. The bioavailability is approximately 50\%, mainly because of gut wall and hepatic first-pass metabolism. Mirtazapine shows linear pharmacokinetics, which is dependent on gender and age: females and the elderly show higher plasma concentrations than males and young adults. The elimination half-life of mirtazapine ranges from 20 to $40 \mathrm{~h}$, which is in agreement with the time to reach steady-state ( 4 to 6 days). Biotransformation is mainly mediated by the CYP2D6 and CYP3A4, with a lesser extent of CYP1A2 and CYP2B6 (ref. ${ }^{9,18,41}$ ). Although no concentration-effect relationship could be established, it was found that with therapeutic dosages of mirtazapine ( 15 to $45 \mathrm{mg} /$ day), plasma concentrations range on average from 5 to 100 $\mathrm{ng} / \mathrm{mL}\left(\right.$ ref. ${ }^{41}$ ). Wide interindividual variability of serum concentrations at each dose level of mirtazapine have been found. Patients with N-desmethylmirtazapine/mirtazapine ratios less than 0.4 had significantly more side effects than those having higher ratios. Comedications were assessed for drug-drug interaction, and significantly lower $\mathrm{N}$-desmethylmirtazapine/mirtazapine ratios were found under concomitant medications of the antidepressant sertraline and the antipsychotic amisulpride ${ }^{42}$. 
Bupropion: This is a dopamine-norepinephrine reuptake inhibitor. The bioavailability is minimally $87 \%$, maximal blood levels are reached 3-5 h after administration, plasma protein binding is about $84 \%$. Bupropion is extensively metabolized by the liver and has an elimination half-life 8-26 h. Hydroxybupropion, the primary active metabolite (the elimination half-life 17-47 h), is formed by cytochrome CYP2B6. At steady-state, $\mathrm{C}_{\max }$ of hydroxybupropion is 4- to 7-fold higher, compared with this of the parent drug. Threohydrobupropion and erythrohydrobupropion (the elimination half-life 37 and $33 \mathrm{~h}$, respectively), the other active metabolites of bupropion, are formed via nonmicrosomal pathways ${ }^{43}$. Bupropion and metabolites demonstrated linear pharmacokinetics and steady-state concentrations are achieved within 8 days of administration. Genetic polymorphisms in CYP2B6 may cause variability in bupropion pharmacokinetics. Bupropion may be a probe drug for CYP2B6 activity in humans ${ }^{44}$. A curvilinear relationship between antidepressant efficacy and trough plasma bupropion concentrations was found. The increase in plasma bupropion concentration from trough level to the $4 \mathrm{~h}$ postdose peak level was also positively related to antidepressant response $\mathrm{e}^{45}$. Plasma levels of bupropion and metabolites, particularly hydroxybupropion, may also predict acute antidepressant response in depressed youths taking bupropion ${ }^{46}$.

\section{Serotonin antagonists and reuptake inhibitors}

Trazodone is a main antidepressant from the serotonin antagonists and reuptake inhibitors group.

Trazodone: Absorption of trazodone is rapid and complete after oral administration and the absolute bioavailability has been reported as $>60 \%$. It is $89-95 \%$ protein bound and achieves a maximum plasma concentration approximately 1-4 $\mathrm{h}$ after dosing. Trazodone is nearly completely metabolized hepatically by hydroxylation, dealkylation, and N-oxidation. $20 \%$ of a trazodone dose undergoes N-dealkylation via CYP3A4 to a pharmacologi- cally active metabolite, m-chlorophenylpiperazine. The active metabolite is further metabolized via CYP2D6, and is therefore subject to CYP2D6 polymorphism. Trazodone showes linear pharmacokinetics and exhibits biphasic elimination with a mean distribution half-life of 3-6 h and an elimination half-life of 5-9 h ( ref $\left.^{47}\right)$. A significant correlation was found between steady-state plasma trazodone levels and its antidepressant efficacy, but not between plasma drug levels and the incidence of side effects ${ }^{48,49}$.

The major reason to use TDM for the guidance of psychopharmacotherapy is the considerable interindividual variability in the pharmacokinetic response of the patient. To produce its characteristic effects, a drug must be present in appropriate concentrations at its sites of action. The latter is not only a function of the dose administered. At the very same dose of psychotropic drugs, a more than 20fold interindividual variation in the medication's steadystate concentration in the body may result, as patients differ in their ability to absorb, distribute, metabolize and excrete drugs due to concurrent disease, age, gender, smoking or eating habits, concomitant medication or genetic peculiarities ${ }^{9,50}$. Considerable and clinically relevant knowledge has been acquired during the past 30 years on the important role of cytochrome P450 forms, CYP1A2, CYP2D6, CYP2C9, CYP2C19, and CYP3A4/5, in the biotransformation of antidepressants (Table 2). The genetically determined polymorphism of CYP2D6 is of high clinical relevance for antidepressants, which are substrates of this form, including tricyclic antidepressants, some selective serotonin reuptake inhibitors (eg, paroxetine and fluoxetine), and "third-generation" antidepressants (eg, venlafaxine and mirtazapine). Clinically, a poor metabolizer (PM) status may represent a higher risk for adverse effects in patients treated with antidepressants known to be substrates of the deficient enzyme, while ultra-rapid metabolizers (UMs) undergo a higher risk for nonresponse, due to subtherapeutic plasma concentrations ${ }^{51}$. On the other hand, second-generation antidepressants

Table 2. Cytochrome P450 (CYP) forms involved in the metabolism of antidepressant drugs ${ }^{9,18,54}$.

\begin{tabular}{lll}
\hline Drug & CYP & Active metabolite \\
\hline Bupropion & 2B6 & hydroxybupropion, threohydrobupropion, erythrohydrobupropion \\
Citalopram & 2C19, 3A4, 2D6 & $\begin{array}{l}\text { desmethylcitalopram, didesmethylcitalopram, citalopram-N-oxid } \\
\text { none }\end{array}$ \\
Duloxetine & 1A2, 2D6 & desmethylcitalopram, didesmethylcitalopram \\
Fluoxetine & 2D6, 2C9, 2C19, 3A4, 2B6 & norfluoxetine \\
Fluvoxamine & 1A2, 2D6 & fluvoxamine acid \\
Mirtazapine & 3A4, 2D6, 1A2, 2B6 & desmethylmirtazapine \\
Paroxetine & 2D6, 3A4, 1A2, 2C19, 3A5 & none \\
Sertraline & 2B6, 2C19, 3A4, 2D6, 2C9 & desmethylsertraline \\
Trazodone & 3A4, 2D6 & m-chlorophenylpiperazine \\
Venlafaxine & 2D6, 3A4, 2C19 & O-desmethylvenlafaxine, N-desmethylvenlafaxine \\
\hline
\end{tabular}


Table 3. Effect of the newer antidepressant drugs in inhibiting the activity of various cytochrome P450 (CYP) forms ${ }^{18,52}$.

\begin{tabular}{lcccccccc}
\hline CYP & Bupropion & Citalopram & Duloxetine & Fluoxetine & Fluvoxamine & Paroxetine & Sertraline & Venlafaxine \\
\hline 1A2 & - & $-/+$ & - & $-/+$ & +++ & $-/+$ & $-/+$ & - \\
2C9 & - & - & - & ++ & ++ & + & + & - \\
$2 \mathrm{C} 19$ & - & $-/+$ & - & ++ & +++ & + & + & - \\
$2 \mathrm{D} 6$ & ++ & + & ++ & +++ & + & +++ & ++ & + \\
$3 \mathrm{~A} 4$ & - & - & - & ++ & ++ & $-/+$ & $-/+$ & - \\
\hline
\end{tabular}

Symbols: - = no effect; + = weak effect; ++ = moderate effect; +++ = strong effect

Table 4. Levels of recommendation for TDM in psychiatry by Arbeitsgemeinschaft für Neuropsychopharmakologie und Pharmakopsychiatrie ${ }^{9}$.

\begin{tabular}{ll}
\hline Level & Evidence \\
\hline 1: strongly & Reported drug concentrations \\
recommended & are established and \\
& evaluated therapeutic reference \\
& ranges. Controlled clinical trials \\
& have shown beneficial effects \\
& of TDM, reports on decreased \\
& tolerability or intoxications.
\end{tabular}

2: recommended

3: useful

4: potentially useful

to pharmacodynamic effects are
Reported drug concentrations were obtained from plasma concentrations at therapeutically effective doses and related to clinical effects; reports on decreased tolerability or intoxications at "supratherapeutic" plasma concentrations.

\section{Reported drug concentrations} were calculated from plasma concentrations at effective doses obtained from

pharmacokinetic studies.

Plasma concentrations related either not yet available or based on retrospective analysis of TDM data, single case reports or non-systematic clinical experience.

Plasma concentrations do not correlate with clinical effects due to unique pharmacology of the drug,

e. g., irreversible blockade of an enzyme, or dosing can be easily guided by clinical symptoms, e. g., sleep induction by a hypnotic drug.
TDM is recommended for dose titration and for special indications or problem solving.

TDM is useful for special indications or problem solving.

TDM is not recommended for dose titration but may be potentially useful for special indications or problem solving.

Clinical Consequences

At therapeutic plasma concentrations highest probability of response or remission; at "subtherapeutic" plasma concentrations: response rate similar to placebo under acute treatment and risk of relapse under chronic treatment; at "supratherapeutic" plasma concentrations: risk of intolerance or intoxication.

TDM will increase the probability of response in non-responders.

At "subtherapeutic" plasma concentrations: risk of poor response; at "supratherapeutic" plasma concentrations: risk of intolerance or intoxication.

TDM can be used to control whether plasma concentrations are plausible for a given dose, or clinical improvement may be attained by dose increase in nonresponders who display too low plasma concentrations.

TDM should be restricted to special indications. 
Table 5. Recommended therapeutic reference ranges, laboratory alert levels and levels of recommendation for TDM(ref. ${ }^{9}$ ).

\begin{tabular}{lccc}
\hline Drugs + active metabolites & Level & $\begin{array}{c}\text { Therapeutic reference range } \\
(\mathrm{ng} / \mathrm{ml})\end{array}$ & $\begin{array}{c}\text { Laboratory alert level } \\
(\mathrm{ng} / \mathrm{ml})\end{array}$ \\
\hline Bupropion + Hydroxybupropion & 3 & $225-1500$ & 2000 \\
Citalopram & 2 & $50-110$ & 220 \\
Duloxetine & 2 & $30-120$ & 240 \\
Escitalopram & 2 & $15-80$ & 160 \\
Fluoxetine + Norfluoxetine & 2 & $120-500$ & 1000 \\
Fluvoxamine & 2 & $60-230$ & 500 \\
Mirtazapine & 2 & $30-80$ & 160 \\
Paroxetine & 3 & $30-120$ & 240 \\
Sertraline & 2 & $10-150$ & 300 \\
Trazodone & 2 & $700-1000$ & 1200 \\
Venlafaxine + O-Desmethylvenlafaxine & 2 & $100-400$ & 800 \\
\hline
\end{tabular}

Table 6. Ranges of metabolite-to-parent drug concentration ratios ${ }^{9}$.

\begin{tabular}{llc}
\hline Drug & Metabolite & $\begin{array}{c}\text { Ratios of concentrations metabolite/parent drug } \\
(\text { mean - sd - mean }+ \text { sd })\end{array}$ \\
\hline Bupropion & hydroxybupropion & $5-47(24 \mathrm{~h}) ; 6-30(12 \mathrm{~h})$ \\
Citalopram & desmethylcitalopram & $0.31-0.60$ \\
Duloxetine & - & - \\
Escitalopram & desmethylcitalopram & $0.3-1.0$ \\
Fluoxetine & norfluoxetine & $0.7-1.9$ \\
Fluvoxamine & fluvoxamine acid & $0-1.2$ \\
Mirtazapine & desmethylmirtazapine & $0.2-1.2$ \\
Paroxetine & - & - \\
Sertraline & desmethylsertraline & $1.7-3.4$ \\
Trazodone & m-chlorophenylpiperazine & $0.04-0.22$ \\
Venlafaxine & O-desmethylvenlafaxine & CYP2D6: EM/IM 0.3-5.2; PM $\leq 0.3 ; \mathrm{UM}>5.2$ \\
& N-desmethylvenlafaxine & $0.46-1.48$ \\
\hline
\end{tabular}

$\mathrm{EM}=$ extensive metabolizers, $\mathrm{IM}=$ intermediate metabolizers, $\mathrm{PM}=$ poor metabolizers, $\mathrm{UM}=$ ultra-rapid metabolizers

differ in their potential for pharmacokinetic drug interactions (Table 3). Fluoxetine and paroxetine are potent inhibitors of CYP2D6, fluvoxamine markedly inhibits CYP1A2 and CYP2C19. For this reason, clinically relevant interactions may be expected when these antidepressants are coadministered with substrates of the pertinent forms, particularly those with a narrow therapeutic index. Duloxetine and bupropion are moderate inhibitors of CYP2D6, and sertraline may cause significant inhibition of this form, but only at high doses. Citalopram, escitalopram, venlafaxine and mirtazapine are weak or negligible inhibitors of CYP forms in vitro and are less likely than other second-generation antidepressants to interact with co-administered medications ${ }^{18,52}$.

The TDM group of the Arbeitsgemeinschaft für Neuropsychopharmakologie und Pharmakopsychiatrie (AGNP) has published literature-based guidelines for optimal use of TDM in psychiatry and defined 4 levels of recommendation, based on empirical evidence: level 1 - strongly recommended, level 2 - recommended, level 3 - useful, and level 4 - potentially useful. The reference ranges listed in generally those for the primary indication (Tables 4, 5). A number of drugs, however, are recommended for several indications (e.g., antidepressant drugs are also used for the treatment of anxiety) but little information is available on optimum plasma concentrations in these situations. So called the "laboratory alert levels" indicate drug concentrations above the recommended reference range that causes the laboratory to feedback immediately to the prescribing physician. The laboratory alert should lead to dose reduction when the patient exhibits signs of intolerance or toxicity. When the high drug concentration is well tolerated by the patient and if dose reduction bears the risk of symptom exacerbation, the dose should remain unchanged. For a number of psychoactive drugs, metabolites actively contribute to the 
overall clinical effect of the parent compound. For this reason, TDM must include the quantification of active metabolites, e.g., in the case of fluoxetine (norfluoxetine). Analysis of pharmacologically inactive metabolites, however, may give useful information on the metabolic state of the patient or on his/her compliance. Table 6 shows the "normal" ratios of concentrations of metabolites to parent drugs. Calculated ranges contain $68 \%$ of the ratios expected under standard dosages, i. e., ratios within the range of the mean $\pm 1 \mathrm{SD}$ assuming normal distribution. A ratio above or below the "normal ratio" can indicate problems of drug adherence or metabolic abnormalities due to a genetic variation or a drug-drug interaction with co-medications exhibiting enzyme inhibiting or inducing properties. In a patient who is genotyped as a poor or ultrarapid metabolizer the medication should not automatically be replaced by another as the dose can often be adapted, using clinical judgement and TDM. AGNP recommends regular monitoring of plasma concentrations under maintenance therapy, at least every 3-6 months, to prevent relapses and rehospitalizations. The frequency of TDM requests may be increased if patients are known to be non-adherent to the medication or in case of changes of co-medications or of smoking (CYP1A2 substrate such as duloxetine) that affect the pharmacokinetics of the drug. As a rule, trough concentrations are measured, but in some situations peak concentrations would show a better correlation with adverse effects. Blood should be collected after at lest 4 drug elimination half-lives after the start of or a change in dosage. In clinical practice, the appropriate sampling time for most psychoactive drugs is one week after stable daily dosing and immediately before ingestion of the morning dose. For the treatment with antidepressant there is good evidence that clinical non-improvement at week 2 is highly predictive for later response and remission. Especially the absence of early improvement appears to be a highly reliable predictor of later non-response. For dose titration with antidepressant drugs is therefore recommend to include symptom rating by the treating physician at baseline and at week 2 in addition to drug concentration measurements ${ }^{9}$.

\section{CONCLUSION}

In conclusion, TDM of antidepressant drugs is a method specific to clinical pharmacology for monitoring drugs using the measurement of drug serum concentrations followed by interpretation and good cooperation with clinician. It is able to help in personalized medicine in the area of dosing individualization because of rational therapy, minimalization of side effects, decrease in mortality and morbidity and reduction in cost of health care. Phenotyping and genotyping can increase therapeutic drug monitoring to a higher level ${ }^{53}$.

\section{ABBREVIATIONS}

5-HT, 5-Hydroxytryptamine, Serotonin; 5-HTT, Serotonin transporter; AGNP, The TDM group of the Arbeitsgemeinschaft für Neuropsychopharmakologie und Pharmakopsychiatrie;

$\mathrm{C}_{\max }$, Maximum plasma concentration; CYP, Cytochrome P450; EM, Extensive metabolizer; IM, Intermediate metabolizer; MAOIs, Monoamine oxidase inhibitors; NaSSA, Noradrenergic and specific serotonergic antidepressant; ODV, O-desmethylvenlafaxine; PM, poor metabolizer; SARIs, Serotonin antagonists and reuptake inhibitors; S-DCT, S-desmethylcitalopram; SNRIs, Serotonin and norepinephrine reuptake inhibiors; SSRIs, Selective serotonin reuptake inhibitors; TCAs, Tricyclic antidepressants; TDM, Therapeutic drug monitoring; $\mathrm{T}_{\max }$, Time to reach maximum plasma concentration; UM, Ultra-rapid metabolizer; VEN, Venlafaxine.

\section{ACKNOWLEDGEMENT}

Authorship contributions: MG: manuscript writing, final approval; IK: literature search, manuscript writing, tables; RU: tables.

Conflict of interest statement: The authors stated that there are no conflicts of interest regarding the publication of this article.

\section{REFERENCES}

1. Furukawa TA, Akechi T, Shimodera S, Yamada M, Miki K, Watanabe N Inagaki $\mathrm{M}$, Yonemoto N. Strategic use of new generation antidepressants for depression: SUN $\left(\wedge \_\wedge\right) D$ study protocol. Trials 2011;12:11635.

2. Reis M, Aamo T, Spigset O, Ahlner J. Serum concentrations of antidepressant drugs in a naturalistic setting: compilation based on a large therapeutic drug monitoring database. Ther Drug Monit 2009;31(1):42-56

3. Mann K, Hiemke Ch, Schmidt LG, Bates DW. Appropriateness of Therapeutic Drug Monitoring for Antidepressants in Routine Psychiatric Inpatient Care. Ther Drug Monit 2006;28:83-8.

4. Lundmark J, Bengtsson F, Nordin C, Reis M, Walinder J. Therapeutic drug monitoring of selective serotonin reuptake inhibitors influences clinical dosing strategies and reduces drug costs in depressed elderly patients. Acta Psychiatr Scand 2000;101:354-9.

5. APA Task Force. Tricyclic antidepressants - blood level measurements and clinical outcome: an APA Task Force Report. Am J Psychiatry 1985; 142:155-62.

6. Glassman AH. Antidepressant plasma levels revisited. Int Clin Psychopharmacol 1994;9(Suppl. 2):25-30.

7. Simmons SA, Perry PJ, Rickert ED, Browne JL. Cost-benefit analysis of prospective pharmacokinetic dosing of nortriptyline in depressed inpatients. J Affect Disord 1985; 8:47-53.

8. Preskorn $\mathrm{SH}$, Fast GA. Therapeutic drug monitoring for anti-depressants: efficacy, safety and cost-effectiveness. J Clin Psychiatry 1991;52(Suppl. 6):23-33.

9. Hiemke C, Baumann P, Bergemann N, Conca A, Dietmaier O, Egberts K, Fric M, Gerlach M, Greiner C, Gründer G, Haen E, HavemannReinecke $U$, Jaquenoud Sirot E, Kirchherr H, Laux G, Lutz UC, Messer T, Müller MJ, Pfuhlmann B, Rambeck B, Riederer P, Schoppek B, Stingl J, Uhr M, Ulrich S, Waschgler R, Zernig G. AGNP Consensus Guidelines for Therapeutic Drug Monitoring in Psychiatry: Update 2011. Pharmacopsychiatry 2011; 44:195-235.

10. Huffman JC, Alpert JE. An approach to the psychopharmacologic care of patients: antidepressants, antipsychotics, anxiolytics, mood 
stabilizers, and natural remedies. Med Clin North Am 2010;94:114160.

11. Andrews PW, Anderson Thomson J, Amstadter A, Neale MC. Primum non nocere: an evolutionary analysis of whether antidepressants do more harm than good. Fron Psychol 2012;3:1-19.

12. Rasmussen $\mathrm{BB}$, Brøsen $\mathrm{K}$. Is therapeutic drug monitoring a case for optimizing clinical outcome and avoiding interactions of the selective serotonin reuptake inhibitors? Ther Drug Monit 2000;22:143-54

13. Prapotnik M, Waschgler R, König P, Moll W, Conca A. Therapeutic drug monitoring of trazodone: are there pharmacokinetic interactions involving citalopram and fluoxetine? Int J Clin Pharmacol Ther 2004;42(2):120-4.

14. Yu BN, Chen GL, He N, Ouyang DS, Chen XP, Liu ZQ, Zhou HH. Pharmacokinetics of citalopram in relation to genetic polymorphism of CYP2C19. Drug Metab Dispos 2003;31:1255-9.

15. Le Bloc'h Y, Woggon B, Weissenrieder H, Brawand-Amey M, Spagnoli J, Eap CB, Baumann P. Routine therapeutic drug monitoring in patients treated with 10-360 mg/day citalopram. Ther Drug Monit 2003;25(5):600-8.

16. Haji EO, Tadić A, Wagner S, Dragicevic A, Müller MJ, Boland K, Rao ML, Fric M, Laux G, Hiemke Ch. Association between citalopram serum levels and clinical improvement of patients with major depression. J Clin Psychopharmacol 2011;31:281-6.

17. Rao N. The clinical pharmacokinetics of escitalopram. Clin Pharmacokinet 2007;46(4):281-90.

18. Spina E, Santoro V, D'Arrigo C. Clinically relevant pharmacokinetic drug interactions with second-generation antidepressants: an update. Clin Ther 2008;30(7):1206-27.

19. Altamura AC, Moro AR, Percudani M. Clinical pharmacokinetics of fluoxetine. Clin Pharmacokinet 1994;26(3):201-14.

20. Gerstenberg G, Aoshima T, Fukasawa T, Yoshida K, Takahashi H, Higuchi H, Murata Y, Shimoyama R, Ohkubo T, Shimizu T, Otan K. Relationship between clinical effects of fluvoxamine and the steady-state plasma concentrations of fluvoxamine and its major metabolite fluvoxamino acid in Japanese depressed patients. Psychopharmacology (Berl). 2003;167(4):443-8.

21. Hiemke C. Paroxetine: pharmacokinetics and pharmacodynamics. Fortschr Neurol Psychiatr 1994;62(Suppl.1):2-8.

22. Jornil J, Jensen KG, Larsen F, Linnet K. Identification of cytochrome P450 isoforms involved in the metabolism of paroxetine and estimation of their importance for human paroxetine metabolism using a population-based simulator. Drug Metab Dispos 2010;38:376-85.

23. Bourin M, Chue P, Guillon Y. Paroxetine: a review. CNS Drug Rev 2001;7(1):25-47.

24. Gex-Fabry M, Eap CB, Oneda B, Gervasoni N, Aubry JM, Bondolfi $G$, Bertschy G. CYP2D6 and ABCB1 genetic variability: influence on paroxetine plasma level and therapeutic response. Ther Drug Monit 2008;30(4):474-82.

25. Hegerl U, Bottlender R, Gallinat J, Kuss HJ, Ackenheil M, Möller HJ. The serotonin syndrome scale: first results on validity. Eur Arch Psy Clin N 1998;248:96-103.

26. Obach RS, Cox LM, Tremaine LM. Sertraline is metabolized by multiple cytochrome P450 enzymes, monoamine oxidases, and glucuronyl transferases in human: an in vitro study. Drug Metab Dispos 2005;33(2):262-70

27. Reis $M$, Aberg-Wistedt $A$, Agren $H$, Höglund $P$, Akerblad AC, Bengtsson F. Serum disposition of sertraline, $\mathrm{N}$-desmethylsertraline and paroxetine: a pharmacokinetic evaluation of repeated drug concentration measurements during 6 months of treatment for major depression. Hum Psychopharmacol 2004;19(5):283-91.

28. Gupta RN, Dziurdzy SA. Therapeutic monitoring of sertraline. Clin Chem 1994;40:496-9.

29. Lundmark J, Reis M, Bengtsson F. Therapeutic drug monitoring of sertraline: variability factors as displayed in a clinical setting. Ther Drug Monit 2000;22(4):446-54.

30. Mauri MC, Fiorentini A, Cerveri G, Volonteri LS, Regispani F, Malvini L, Boscati L, Baido RL, Invernizzi G. Long-term efficacy and therapeutic drug monitoring of sertraline in major depression. Hum Psychopharmacol 2003;18(5):385-8.

31. Shams ME, Arneth B, Hiemke C, Dragicevic A, Müller MJ, Kaiser R, Lackner K, Härtter S. CYP2D6 polymorphism and clinical effect of the antidepressant venlafaxine. J Clin Pharm Ther 2006;31(5):493-502.

32. Nichols Al, Lobello K, Guico-Pabia CJ, Paul J, Preskorn SH. Venlafaxine metabolism as a marker of cytochrome P450 enzyme 2D6 metabolizer status. J Clin Psychopharmacol 2009; 29(4):383-6.
33. Unterecker S, Hiemke C, Greiner C, Haen E, Jabs B, Deckert J, Pfuhlmann B. The Effect of Age, Sex, Smoking and Co-Medication on Serum Levels of Venlafaxine and O-Desmethylvenlafaxine under Naturalistic Conditions. Pharmacopsychiatry 2012;45(6):229-35.

34. Charlier C, Pinto E, Ansseau M, Plomteux G. Venlafaxine: the relationship between dose, plasma concentration and clinical response in depressive patients. J Psychopharmacol 2002;16(4):369-72.

35. Gex-Fabry M, Balant-Gorgia AE, Balant LP, Rudaz S, Veuthey JL, Bertschy G. Time course of clinical response to venlafaxine: relevance of plasma level and chirality. Eur J Clin Pharmacol. 2004; 59(12):883-91.

36. Grundmann M, Urinovska R, Silhan P, Kacirova I. Venlafaxine intoxication - importance of TDM, phenotyping and genotyping. Klin Farmakol Farm 2012;26(2):90-2.

37. Shams ME, Arneth B, Hiemke C, Dragicevic A, Müller MJ, Kaiser R, Lackner K, Härtter S. CYP2D6 polymorphism and clinical effect of the antidepressant venlafaxine. J Clin Pharm Ther 2006;31(5):493-502.

38. Carter NJ, McCormack PL. Duloxetine: a review of its use in the treatment of generalized anxiety disorder. CNS Drugs. 2009;23(6):523-41.

39. Knadler MP, Lobo E, Chappell J, Bergstrom R. Duloxetine: clinical pharmacokinetics and drug interactions. Clin Pharmacokinet 2011;50(5):281-94.

40. Waldschmitt C, Vogel F, Pfuhlmann B, Hiemke C. Duloxetine serum concentrations and clinical effects. Data from a therapeutic drug monitoring (TDM) survey. Pharmacopsychiatry 2009;42(5):189-93.

41. Timmer CJ, Sitsen JM, Delbressine LP. Clinical pharmacokinetics of mirtazapine. Clin Pharmacokinet 2000 Jun;38(6):461-74.

42. Shams M, Hiemke Ch, Hartter S. Therapeutic Drug Monitoring of the Antidepressant Mirtazapine and Its N-Demethylated Metabolite in Human Serum. Ther Drug Monit 2004;26:78-84.

43. Jefferson JW, Pradko JF, Muir KT. Bupropion for major depressive disorder: Pharmacokinetic and formulation considerations.Clin Ther 2005;27(11):1685-95.

44. Faucette SR, Hawke RL, Lecluyse EL, Shord SS, Yan B, Laethem RM Lindley $\mathrm{CM}$. Validation of bupropion hydroxylation as a selective marker of human cytochrome P450 2B6 catalytic activity. Drug Metab Dispos 2000;28(10):1222-30.

45. Preskorn SH. Antidepressant response and plasma concentrations of bupropion. J Clin Psychiatry 1983;44(5 Pt 2):137-9.

46. Daviss WB, Perel JM, Brent DA, Axelson DA, Rudolph GR, Gilchrist $R$, Nuss $S$, Birmaher B. Acute antidepressant response and plasma levels of bupropion and metabolites in a pediatric-aged sample: an exploratory study. Ther Drug Monit 2006;28(2):190-8.

47. Karhu D, Gossen ER, Mostert A, Cronjé T, Fradette C. Safety, tolerability, and pharmacokinetics of once-daily trazodone extended-release caplets in healthy subjects. Int J Clin Pharmacol Ther 2011;49(12):730-43.

48. Monteleone P, Gnocchi G. Evidence for a linear relationship between plasma trazodone levels and clinical response in depression in the elderly. Clin Neuropharmacol 1990;13(Suppl 1):84-9.

49. Mihara K, Yasui-Furukori N, Kondo T, Ishida M, Ono S, Ohkubo T, Osanai T, Sugawara K, Otani K, Kaneko S. Relationship between plasma concentrations of trazodone and its active metabolite, $\mathrm{m}$ chlorophenylpiperazine, and its clinical effect in depressed patients. Ther Drug Monit 2002;24(4):563-6.

50. Hiemke Ch. Therapeutic drug monitoring in neuropsychopharmacology: does it hold its promises? Eur Arch Psychiatry Clin Neurosci 2008;258(Suppl 1):21-7.

51. Baumann P, Ulrich S, Eckermann G, Gerlach M, Kuss HJ, Laux G, Müller-Oerlinghausen B, Rao ML, Riederer P, Zernig G, Hiemke C; Arbeitsgemeinschaft fur Neuropsychopharmakologie und Pharmakopsyhiatrie - Therapeutic Drug Monitoring group. The AGNP-TDM Expert Group Consensus Guidelines: focus on therapeutic monitoring of antidepressants. Dialogues Clin Neurosci 2005;7:231-47.

52. Mitchell PB. Therapeutic drug monitoring of psychotropic medications. Br J Clin Pharmacol 2000;49:303-12.

53. Grundmann M, Kacirova I. Significance of TDM, phenotyping and genotyping for the correct drug dosage. Cas Lek Ces 2010;149:482-7.

54. Tekes K, Hashemi F, Szegi P, Sotonyi P, Laufer R, Kalasz H. Prodrugs and active metabolites among antidepressive compounds. Neuropsychopharmacol Hung 2011;12(2):103-10. 